\title{
Analysis of TBC1D4 in patients with severe insulin resistance
}

\author{
S. Dash • C. Langenberg • K. A. Fawcett • R. K. Semple • S. Romeo • S. Sharp • \\ H. Sano • G. E. Lienhard • J. J. Rochford • T. Howlett • A. F. Massoud • \\ P. Hindmarsh • S. J. Howell • R. J. Wilkinson • V. Lyssenko • L. Groop • M. G. Baroni • \\ I. Barroso • N. J. Wareham • S. O' Rahilly • D. B. Savage
}

Received: 23 October 2009 / Accepted: 18 February 2010 / Published online: 27 March 2010

(C) The Author(s) 2010. This article is published with open access at Springerlink.com

Keywords AS160 - Diabetes · Genetics · GLUT4 .

Insulin resistance $\cdot T B C 1 D 4$

\section{Abbreviations}

MSH Melanocyte-stimulating hormone

SIR Severe insulin resistance

TBC1D4 Tre-2 BUB2 CDC16, 1 domain family member 4
To the Editor: Glucose uptake into muscle and fat is impaired in insulin-resistant states [1]. In response to insulin, vesicles containing GLUT4 are redistributed from the cell interior to the plasma membrane where they dock and fuse with the plasma membrane (GLUT4 translocation), enabling glucose uptake [2]. In vitro studies suggest that Tre-2 BUB2 CDC16, 1 domain family member 4 (TBC1D4),

Electronic supplementary material The online version of this article (doi:10.1007/s00125-010-1724-x) contains supplementary material, which is available to authorised users.

S. Dash • R. K. Semple $\cdot$ S. Romeo · J. J. Rochford •

H. Sano $\cdot$ G. E. Lienhard

S. O’ Rahilly $(\bowtie) \cdot$ D. B. Savage $(\bowtie)$

University of Cambridge Metabolic Research Laboratories,

Level 4, Institute of Metabolic Science,

Department of Biochemistry,

Dartmouth Medical School,

University of Cambridge,

Addenbrooke's Hospital Box 289, Hills Road,

Cambridge CB2 0QQ, UK

e-mail: so104@medschl.cam.ac.uk

e-mail: dbs23@medschl.cam.ac.uk

C. Langenberg $\cdot$ S. Sharp $\cdot$ N. J. Wareham

MRC Epidemiology Unit,

Institute of Metabolic Sciences, Addenbrooke's Hospital,

Cambridge, UK

\section{K. A. Fawcett $\cdot$ I. Barroso}

Metabolic Disease Group, Wellcome Trust Sanger Institute,

Wellcome Trust Genome Campus,

Hinxton, Cambridgeshire, UK

V. Lyssenko • L. Groop

Department of Clinical Sciences, Lund University,

Malmö, Sweden

Hanover, NH, USA

\section{R. J. Wilkinson}

Division of Medicine, Imperial College,

London, UK

A. F. Massoud

Children's Services,

Northwick Park Hospital,

Harrow, Middlesex, UK

P. Hindmarsh

London Centre for Paediatric Endocrinology and Metabolism,

Cobbold Laboratories, Middlesex Hospital,

London, UK

T. Howlett

Department of Diabetes and Endocrinology,

Leicester Royal Infirmary,

Leicester, UK

M. G. Baroni

Department of Medical Sciences, Endocrinology and Metabolism,

University of Cagliari,

Cagliari, Sardinia, Italy

S. J. Howell

Department of Diabetes and Endocrinology,

Royal Preston Hospital,

Preston, UK 
which is also known as Akt substrate of $160 \mathrm{kDa}$ (AS160), plays an important role in GLUT4 translocation [3] making $T B C 1 D 4$ an excellent candidate gene for insulin resistance.

We have assembled a cohort of patients with severe insulin resistance (SIR) (diagnosed on the basis of a fasting insulin of $>150 \mathrm{pmol} / 1$ and/or a postprandial insulin of $>1500 \mathrm{pmol} / 1$ and/or the presence of acanthosis nigricans or, if diabetic, a requirement of $>200 \mathrm{U}$ insulin daily) likely to be enriched for monogenic causes of the condition. All participants provided informed consent following approval by our local ethics committee. We recently reported a novel pathogenic mutation in TBC1D4 (R363X) which impaired GLUT4 translocation [4]. It is associated with an unusual insulin-resistance phenotype characterised by normal fasting insulin and glucose levels with elevated postprandial glucose and a disproportionate rise in insulin following glucose ingestion [4]. In the fasting state, low circulating insulin levels predominantly regulate hepatic glucose production. After a glucose challenge, insulin levels rise, suppressing hepatic glucose production and promoting glucose uptake in muscle and fat, suggesting that this phenotype may be indicative of peripheral insulin resistance with preserved hepatic insulin sensitivity [4]. In order to investigate whether mutations in TBC1D4 contribute more widely to SIR, we sequenced the coding regions of TBC1D4 (21 exons, 3894 nucleotides) in 156 patients from the cohort. Here we describe three additional novel nonsynonymous $T B C 1 D 4$ variants.

An N1206S variant (c.3617 G>A) results in the substitution of an asparagine residue (conserved from humans to zebrafish) with serine. This heterozygous variant, predicted to be 'probably damaging' by PolyPhen [5] and 'deleterious' by SIFT [6], was found in two patients. The first patient presented at age 16 years with weight gain (BMI $28.5 \mathrm{~kg} / \mathrm{m}^{2}$, BMI SD score $+2.1 \mathrm{~kg} / \mathrm{m}^{2}$, acanthosis nigricans and normal fasting glucose and insulin levels; see Electronic supplementary material [ESM] Table 1). During an OGTT she had impaired glucose tolerance with a dramatic rise in insulin (ratio of peak to fasting insulin $=90$, mean ratio in BMI-matched controls in the Ely study 8.5) [7]. Her mother (BMI $25 \mathrm{~kg} / \mathrm{m}^{2}$, BMI SD score $+1 \mathrm{~kg} / \mathrm{m}^{2}$ ), the only accessible family member with the variant, was normoglycaemic but had a modestly elevated peak-tofasting-insulin ratio of 10.5 (ESM Table 1) suggesting that this variant alone could not fully account for the proband's phenotype. The second SIR patient with this variant was a woman with poorly controlled type 2 diabetes mellitus despite administration of nearly $200 \mathrm{U}$ insulin. It was not possible to assess her family members.

The N1206S variant was present in $1.6 \%$ of 200 ethnically matched healthy controls so we went on to evaluate its effect in three separate population studies with OGTT data. The Ely study is a population-based study comprising 1,669 participants [7]. The Botnia study is a prospective study of 2,770 Finns followed up for a median period of 23.5 years [8]. The third population was a cohort of 524 unrelated morbidly obese Italians (mean BMI of $41 \mathrm{~kg} / \mathrm{m}^{2}$ ) recruited from the Department of Clinical Sciences, University of Rome, Rome, Italy [9]. Our hypothesis was that N1206S carriers would have elevated $2 \mathrm{~h}$ glucose levels and a disproportionate rise in insulin post OGTT, as judged by higher ratios of insulin at 60 and $120 \mathrm{~min}$ to fasting insulin. The combined minor allele frequency was $1.2 \%$ in the three populations. Pooled estimates from inverse-variance fixed-effects meta-analyses of 4468 individuals across the three studies (Table 1) indicate that individuals with the N1206S variant have higher $120 \mathrm{~min}$ blood glucose levels $(p=0.021)$ and ratios of 60 and $120 \mathrm{~min}$ to fasting insulin $(p=0.006$ and $p=0.024$, respectively). There were no significant differences in fasting glucose and insulin levels. This suggests that participants with the N1206S variant may have a small but significant impairment in peripheral insulin sensitivity with preserved hepatic insulin sensitivity. There was no statistically significant heterogeneity between the studies. In an effort to characterise the effects of this variant on GLUT4 translocation, we transiently transfected 3T3L1 adipocytes with wild-type or N1206S TBCID4 cDNA vectors as described previously [4], but were unable to demonstrate any differences in GLUT4 translocation (ESM Fig. 1). This may reflect the apparently subtle effects of this mutant in vivo or a lack of sensitivity of the in vitro assay.

A heterozygous N655Y variant (c. 1964T>A; PolyPhen prediction: 'probably damaging'; SIFT prediction: 'deleterious') $[5,6]$ causes the substitution of an asparagine residue (conserved in a range of species from humans to mice) with tyrosine at amino acid number 655 . It was found in a SIR patient with morbid obesity since childhood and was absent in 200 ethnically matched controls. The proband also has a pathogenic Y221C mutation in the beta melanocyte-stimulating hormone $(\beta \mathrm{MSH})$ region of the $P O M C$ gene, which is likely to contribute to her obesity (current BMI $45 \mathrm{~kg} / \mathrm{m}^{2}$, BMI SD score $\left.+3.9 \mathrm{~kg} / \mathrm{m}^{2}\right)$ (POMC encodes pro-opiomelanocortin) [10]. She was normoglycaemic with elevated fasting and postprandial insulin levels compared with sex- and BMI-matched controls from the Ely study (ESM Table 1) [7]. Her mother (BMI $34 \mathrm{~kg} / \mathrm{m}^{2}$, BMI SD score $+2.7 \mathrm{~kg} / \mathrm{m}^{2}$ ) was the only accessible family member. She too had the N655Y TBC1D4 and the Y221C $P O M C$ variant. Although her fasting and postprandial insulin levels, in isolation, were compatible with her BMI, she had an elevated peak-to-fasting-insulin ratio of 13 (ESM Table 1). Although the proband's severe insulin resistance can be explained by her morbid obesity, it is possible that the $\mathrm{N} 655 \mathrm{Y}$ variant might also be contributing to her phenotype. However, the variant had no effects on 


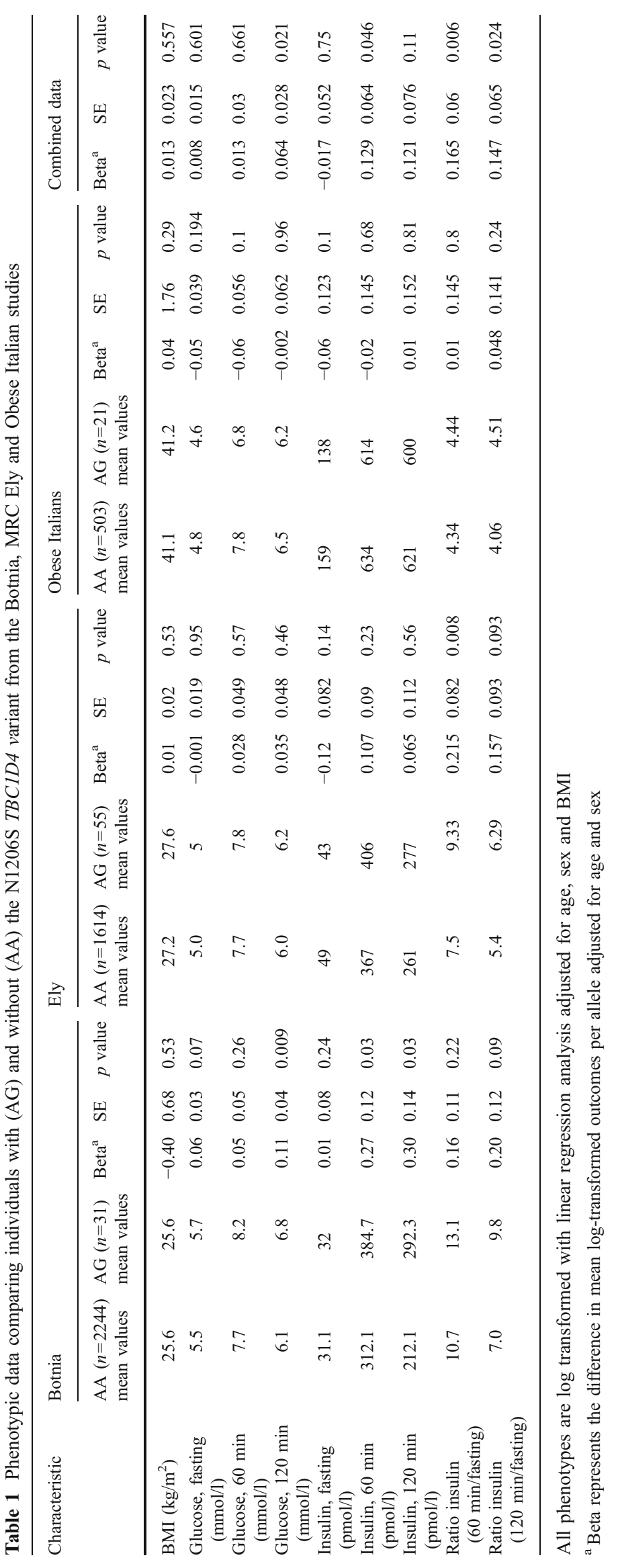


GLUT4 translocation in transfected 3T3L1 adipocytes (ESM Fig. 1).

An N785K variant (c. $2355 \mathrm{G}>\mathrm{C}$; PolyPhen prediction: 'benign'; SIFT prediction: 'tolerated') [5,6] was identified in two SIR patients of Pakistani origin. This variant causes the substitution of a non-conserved asparagine residue with lysine at amino acid number 785 . It was present in one out of 192 ethnically matched control alleles. The first patient (BMI $24.1 \mathrm{~kg} / \mathrm{m}^{2}$, BMI SD score $+1.58 \mathrm{~kg} / \mathrm{m}^{2}$, aged 15 ) with the variant was a young man diagnosed with diabetes at the age of 3 years who had poor glycaemic control despite being on almost $200 \mathrm{U}$ insulin per day and metformin. The other patient presented with acanthosis nigricans during puberty following a period of weight gain. She had a BMI of $33.5 \mathrm{~kg} / \mathrm{m}^{2}$ (BMI SD score $2.9 \mathrm{~kg} / \mathrm{m}^{2}$ ) at the age of 16 with normoglycaemia and elevated fasting and postprandial insulin levels adjusted for BMI (ESM Table 1) [7]. Both probands and their families declined further assessment. This variant was not studied in vitro.

In summary, we have described three novel nonsynonymous variants in TBC1D4. One of these, the N1206S polymorphism, is associated with higher $2 \mathrm{~h}$ glucose levels and a greater postprandial rise in insulin, which might be indicative of isolated peripheral insulin resistance. However, we acknowledge that the effect size is small and that further follow-up studies are needed to confirm this. Because of the lack of adequate co-segregation data, it was not possible to convincingly establish a pathogenic role for the $\mathrm{N} 655 \mathrm{Y}$ and $\mathrm{N} 785 \mathrm{~K}$ variants.

Acknowledgements The authors would like to thank all the patients and volunteers who participated in the studies. These studies were funded by the Medical Research Council (S. Dash, C. Langenberg, S. Sharp, J. J. Rochford, R. J. Wilkinson, N. J. Wareham), Wellcome Trust (R. K. Semple, K. A. Fawcett, I. Barroso, S. O’ Rahilly, D. B. Savage), GlaxoSmithKline (D. B. Savage), Raymond \& Beverly Sackler scholarship (S. Dash), the National Institute for Health
Research Cambridge Biomedical Research Centre and Grant DK25336 from the National Institutes of Health (to G. E. Lienhard).

Duality of interest The authors declare that there is no duality of interest associated with this manuscript.

Open Access This article is distributed under the terms of the Creative Commons Attribution Noncommercial License which permits any noncommercial use, distribution, and reproduction in any medium, provided the original author(s) and source are credited.

\section{References}

1. Savage DB, Petersen KF, Shulman GI (2007) Disordered lipid metabolism and the pathogenesis of insulin resistance. Physiol Rev 87:507-520

2. Huang S, Czech MP (2007) The GLUT4 glucose transporter. Cell Metab 5:237-252

3. Sakamoto K, Holman GD (2008) Emerging role for AS160/ TBC1D4 and TBC1D1 in the regulation of GLUT4 traffic. Am J Physiol Endocrinol Metab 295:E29-E37

4. Dash S, Sano H, Rochford JJ et al (2009) A truncation mutation in TBC1D4 in a family with acanthosis nigricans and postprandial hyperinsulinemia. Proc Natl Acad Sci U S A 106:9350-9355

5. PolyPhen: prediction of functional effect of human nsSNPs. Available from http://genetics.bwh.harvard.edu/pph/, accessed 1 October 2009

6. J. Craig Venter Institute. SIFT. Available from http://sift.jcvi.org, accessed 1 October 2009

7. Loos RJ, Franks PW, Francis RW et al (2007) TCF7L2 polymorphisms modulate proinsulin levels and beta-cell function in a British Europid population. Diabetes 56:1943-1947

8. Lyssenko V, Jonsson A, Almgren P et al (2008) Clinical risk factors, DNA variants, and the development of type 2 diabetes. N Engl J Med 359:2220-2232

9. Romeo S, Sentinelli F, Cavallo MG et al (2008) Search for genetic variants of the SYNTAXIN 1A (STX1A) gene: the $-352 \mathrm{~A}>\mathrm{T}$ variant in the STX1A promoter associates with impaired glucose metabolism in an Italian obese population. Int J Obes (Lond) 32:413-420

10. Lee YS, Challis BG, Thompson DA et al (2006) A POMC variant implicates beta-melanocyte-stimulating hormone in the control of human energy balance. Cell Metab 3:135-140 